\title{
NONRESONANCE CONDITIONS ON THE POTENTIAL FOR A SECOND-ORDER PERIODIC BOUNDARY VALUE PROBLEM
}

\author{
PIERPAOLO OMARI AND FABIO ZANOLIN \\ (Communicated by Charles C. Pugh)
}

ABSTRACT. We consider the periodic problem

$$
\begin{gathered}
-u^{\prime \prime}=f(u)+h(t), \\
u(0)=u(2 \pi), \quad u^{\prime}(0)=u^{\prime}(2 \pi),
\end{gathered}
$$

and prove its solvability for any given $h$, under new assumptions on the asymptotic behaviour of the potential of the nonlinearity $f$, with respect to two consecutive eigenvalues of the associated linear problem.

\section{INTRODUCTION AND STATEMENTS}

In this paper we are concerned with the solvability of the periodic problem

$$
\begin{gathered}
-u^{\prime \prime}=f(u)+h(t), \\
u(0)=u(2 \pi), \quad u^{\prime}(0)=u^{\prime}(2 \pi),
\end{gathered}
$$

where $f$ is a continuous real-valued function, $h \in L^{1}(0,2 \pi)$, and solutions are intended in the Caratheodory sense. Here, we consider the case, extensively discussed in the literature, where the nonlinearity $f$ lies asymptotically between two consecutive higher eigenvalues of the linear operator $-d^{2} / d t^{2}$, with periodic boundary conditions on $[0,2 \pi]$. Precisely, we suppose that

$$
\begin{aligned}
& \text { for some integer } N \geq 1, \\
& \qquad N^{2} \leq \liminf _{s \rightarrow \pm \infty} f(s) / s \leq \limsup _{s \rightarrow \pm \infty} f(s) / s \leq(N+1)^{2} .
\end{aligned}
$$

As is well known, this assumption is not sufficient to ensure nonresonance, i.e., the existence of a solution to (1.1) for any given $h$. Conversely, if $u$ denotes a solution of (1.1), subtracting from both sides of the equation $N^{2} u$ (respectively, $(N+1)^{2} u$ ), multiplying by an eigenfunction corresponding to the eigenvalue $N^{2}$ (respectively, $\left.(N+1)^{2}\right)$, and integrating, one sees that a necessary condition for nonresonance is that both functions $f(s)-N^{2} s$ and $(N+1)^{2} s-f(s)$ be unbounded on $\mathbb{R}$. Yet a recent result [DIZ, Theorem 5.2] shows that even the strengthened form of unboundedness

$$
N^{2}<\limsup _{s \rightarrow \pm \infty} f(s) / s \text { and } \liminf _{s \rightarrow \pm \infty} f(s) / s<(N+1)^{2}
$$

Received by the editors May 14, 1991.

1991 Mathematics Subject Classification. Primary 34B15, 34C25. 
is not sufficient, together with $\left(f_{1}\right)$, to yield nonresonance. Indeed, according to [DIZ], for any integer $M \geq 1$, there exists a nonlinear map $f$, with

$$
A=\liminf _{s \rightarrow \pm \infty} f(s) / s<\limsup _{s \rightarrow \pm \infty} f(s) / s=B
$$

and $M^{2} \in[A, B]$, such that (1.1) has no solution, for some smooth function $h$.

On the other hand, the inequalities

$$
\liminf _{s \rightarrow \pm \infty} f(s) / s \leq \liminf _{s \rightarrow \pm \infty} 2 F(s) / s^{2} \leq \limsup _{s \rightarrow \pm \infty} 2 F(s) / s^{2} \leq \limsup _{s \rightarrow \pm \infty} f(s) / s,
$$

where $F(s)=\int_{[0, s]} f(\xi) d \xi$, naturally lead one to introduce the following condition, stricter than $\left(\mathrm{f}_{2}\right)$,

$$
N^{2}<\limsup _{s \rightarrow \pm \infty} 2 F(s) / s^{2} \text { and } \liminf _{s \rightarrow \pm \infty} 2 F(s) / s^{2}<(N+1)^{2}
$$

and to ask whether it yields nonresonance, when is coupled with $\left(f_{1}\right)$. A positive answer is provided by the following theorem, where it is also shown that $\left(F_{0}\right)$ can be slightly weakened, requiring that the inequalities be satisfied only at $+\infty$ or at $-\infty$. (Technically, this exploits the fact that eigenfunctions corresponding to nonzero eigenvalues change sign on $[0,2 \pi]$.)

Theorem. Assume $\left(\mathrm{f}_{1}\right)$ and suppose that at least one of the following conditions holds

$$
\begin{array}{lll}
N^{2}<\limsup _{s \rightarrow+\infty} 2 F(s) / s^{2} & \text { and } & \liminf _{s \rightarrow+\infty} 2 F(s) / s^{2}<(N+1)^{2}, \\
N^{2}<\limsup _{s \rightarrow-\infty} 2 F(s) / s^{2} & \text { and } & \liminf _{s \rightarrow-\infty} 2 F(s) / s^{2}<(N+1)^{2}, \\
N^{2}<\limsup _{s \rightarrow+\infty} 2 F(s) / s^{2} & \text { and } & \liminf _{s \rightarrow-\infty} 2 F(s) / s^{2}<(N+1)^{2}, \\
N^{2}<\limsup _{s \rightarrow-\infty} 2 F(s) / s^{2} & \text { and } & \liminf _{s \rightarrow+\infty} 2 F(s) / s^{2}<(N+1)^{2} .
\end{array}
$$

Then problem (1.1) has at least one solution for any given $h \in L^{1}(0,2 \pi)$.

We recall that conditions $\left(f_{1}\right)$ and

$$
N^{2}<\liminf _{s \rightarrow \pm \infty} 2 F(s) / s^{2} \text { and } \limsup _{s \rightarrow \pm \infty} 2 F(s) / s^{2}<(N+1)^{2}
$$

have been recently considered in [CO] in the context of elliptic equations, and they turn out to be equivalent (cf. [GO2, Appendix]) to certain density conditions first introduced in [DFG]. Of course, $\left(\mathrm{F}_{0}\right)$ and therefore each $\left(\mathrm{F}_{i}\right)$, $i=1, \ldots, 4$, are weaker than $\left(\mathrm{F}_{5}\right)$; the following example shows that this is still true when $\left(f_{1}\right)$ is assumed as well.

Example. Let $\left\{a_{n}\right\}$ and $\left\{b_{n}\right\}$ be two increasing sequences of real numbers, with

$$
0<a_{n}<b_{n}=a_{n+1}-1
$$

for every $n \in \mathbb{N}$. Suppose also that $\left\{a_{n}\right\}$ satisfies the recursive relation

$$
a_{0}=0 \text { and } a_{n+1} \geq a_{n}^{2}+2 .
$$

Let $g: \mathbb{R} \rightarrow \mathbb{R}$ be any continuous function such that

$$
|g(s) / s| \leq 1
$$


for every $s \neq 0$ and

$$
g(s)= \begin{cases}s & \text { for } a_{n} \leq s \leq b_{n}, n \text { even, } \\ -s & \text { for } a_{n} \leq s \leq b_{n}, n \text { odd } .\end{cases}
$$

Setting $G(s)=\int_{[0, s]} g(\xi) d \xi$, we have, for any even integer $n$,

$$
\begin{aligned}
G\left(b_{n}\right) & =\int_{\left[0, a_{n}\right]} g(\xi) d \xi+\int_{\left[a_{n}, b_{n}-1\right]} g(\xi) d \xi+\int_{\left[b_{n}-1, b_{n}\right]} g(\xi) d \xi \\
& \geq-\frac{1}{2} a_{n}^{2}+\frac{1}{2}\left(b_{n}-1\right)^{2}-\frac{1}{2} a_{n}^{2}+b_{n}-1 \\
& =\frac{1}{2}\left(b_{n}-1\right)^{2}-a_{n}^{2}+a_{n+1}-2 \geq \frac{1}{2}\left(b_{n}-1\right)^{2} .
\end{aligned}
$$

Hence, we conclude that

$$
\limsup _{s \rightarrow+\infty} 2 G(s) / s^{2}=1 \quad\left(=\limsup _{|s| \rightarrow+\infty} g(s) / s\right) .
$$

A similar computation, performed on $G\left(b_{n}\right)$, with $n$ odd, yields

$$
\liminf _{s \rightarrow+\infty} 2 G(s) / s^{2}=-1 \quad\left(=\liminf _{|s| \rightarrow+\infty} g(s) / s\right) .
$$

Finally, for any fixed integer $N \geq 1$, we set

$$
f(s)=\frac{1}{2}\left(N^{2}+(N+1)^{2}\right) s+\frac{1}{2}(2 N+1) g(s)
$$

for $s \in \mathbb{R}$. Note that no condition is imposed on $f(s)$, for $s<0$, besides $N^{2} \leq f(s) / s \leq(N+1)^{2}$.

Clearly, the function $f$ just defined satisfies condition $\left(\mathrm{f}_{1}\right)$ and $\left(\mathrm{F}_{1}\right)$, but does not satisfy $\left(F_{5}\right)$. Accordingly, we are able to produce the example of a problem to which our result applies, while that in [CO] does not. We also stress that neither the related results given in [ALP, MW1, D, OZ, MW2, GO1, FF, DIZ, DZ, R, Q], can be used here, even if they cover other situations where our theorem may fail.

Finally, we point out that a result similar to that stated above holds as well for the Picard or the Neumann problems associated to the equation in (1.1). Further details in this direction will be given elsewhere.

\section{Proof}

We will prove the theorem under assumptions $\left(f_{1}\right)$ and $\left(F_{1}\right)$ or $\left(f_{1}\right)$ and $\left(\mathrm{F}_{3}\right)$, since in the remaining cases the proof proceeds similarly.

Let us fix a number $\vartheta$, with $N^{2}<\vartheta<(N+1)^{2}$, and let us denote by $H$ the operator that sends any function $e \in L^{1}(0,2 \pi)$ on the unique solution $u \in W^{2,1}(0,2 \pi)$ of the problem

$$
\begin{gathered}
-u^{\prime \prime}-\vartheta u=e(t), \\
u(0)=u(2 \pi), \quad u^{\prime}(0)=u^{\prime}(2 \pi) .
\end{gathered}
$$

Then the solutions of problem $(1.1)$ in $W^{2,1}(0,2 \pi)$ are precisely the solutions in, say, $C^{0}([0,2 \pi])$ of the compact fixed point equation

$$
u=H(f(u)-\vartheta u+h) \text {. }
$$


We will solve (2.1), applying Leray-Schauder degree theory. To this end, we consider the homotopic equation

$$
u=\lambda H(f(u)-\vartheta u+h),
$$

with $\lambda \in[0,1]$, which corresponds to the problem

$$
\begin{aligned}
& -u^{\prime \prime}=(1-\lambda) \vartheta u+\lambda f(u)+\lambda h(t), \\
& u(0)=u(2 \pi), \quad u^{\prime}(0)=u^{\prime}(2 \pi) .
\end{aligned}
$$

Throughout, $u$ will stand for an arbitrary solution of problem (2.2), for some $\lambda \in[0,1]$. Moreover, $P$ and $Q$ will denote, respectively, the orthogonal projections in $L^{2}(0,2 \pi)$ onto the eigenspaces $\operatorname{Span}\{\sin (N t), \cos (N t)\}$ and $\operatorname{Span}\{\sin ((N+1) t), \cos ((N+1) t)\}$, corresponding to the eigenvalues $N^{2}$ and $(N+1)^{2}$. Of course, $P$ and $Q$ can be extended as bounded operators to $L^{1}(0,2 \pi)$. Finally, we will indicate by $|\cdot|_{p}$, with $1 \leq p \leq \infty$ and $\|\cdot\|$ the norms of $L^{p}(0,2 \pi)$ and $W^{2,1}(0,2 \pi)$, respectively.

Step 1. We prove that assumption $\left(f_{1}\right)$ implies that

for every $\varepsilon>0$, there exists a constant $c_{\varepsilon}$ depending only on $\varepsilon$

$$
\text { such that }\|u-P u-Q u\| \leq \varepsilon|u|_{2}+c_{\varepsilon} \text {. }
$$

The equation in (2.2) can be rewritten in the form

$$
-u^{\prime \prime}-N^{2} u=g(t, u, \lambda)+\lambda(h-P h),
$$

where $g(t, s, \lambda)=(1-\lambda) \vartheta s+\lambda f(s)-N^{2} s+\lambda(P h)(t)$, for $t \in[0,2 \pi], s \in \mathbb{R}$, and $\lambda \in[0,1]$. The function $g$ is continuous and satisfies

$$
0 \leq \liminf _{s \rightarrow \pm \infty} g(t, s, \lambda) / s \leq \limsup _{s \rightarrow \pm \infty} g(t, s, \lambda) / s \leq 2 N+1,
$$

uniformly in $t \in[0,2 \pi]$ and $\lambda \in[0,1]$. By elementary computations, one can prove that $(2.5)$ implies that

$$
\begin{aligned}
& \text { for every } \varepsilon>0, \text { there exists } d_{\varepsilon} \text { such that } \\
& \qquad s g(t, s, \lambda) \geq(2 N+1)^{-1}|g(t, s, \lambda)|^{2}-\varepsilon|s|^{2}-d_{\varepsilon},
\end{aligned}
$$

for every $t \in[0,2 \pi], s \in \mathbb{R}$, and $\lambda \in[0,1]$.

Let us denote by $K$ the operator that sends any function $w \in L^{1}(0,2 \pi)$, with $P w=0$, on the unique solution $z \in W^{2,1}(0,2 \pi)$, with $P z=0$, of the problem

$$
\begin{gathered}
-z^{\prime \prime}-N^{2} z=w(t), \\
z(0)=z(2 \pi), \quad z^{\prime}(0)=z^{\prime}(2 \pi) .
\end{gathered}
$$

Of course, $K$ is bounded from $L^{1}(0,2 \pi) \cap \operatorname{ker} P$ to $W^{2,1}(0,2 \pi)$. Then we observe that $P g(\cdot, u, \lambda)=0$ and multiply the equation in (2.4) by $K g(\cdot, u, \lambda)$. Integration by parts and the use of the boundary conditions give

$$
\langle u, g(\cdot, u, \lambda)\rangle=\langle g(\cdot, u, \lambda), K g(\cdot, u, \lambda)\rangle+\lambda\langle(I-P) h, K g(\cdot, u, \lambda)\rangle,
$$


where $\langle\cdot, \cdot\rangle$ stands for the $L^{2}$-bilinear pairing and $I$ denotes the identity operator. Hence, using (2.6) and $K Q=Q K$, we easily obtain

$$
\begin{aligned}
(2 N & +1)^{-1}|Q g(\cdot, u, \lambda)|_{2}^{2}+(2 N+1)^{-1}|(I-Q) g(\cdot, u, \lambda)|_{2}^{2}-\varepsilon|u|_{2}^{2}-2 \pi d_{\varepsilon} \\
\leq & \langle g(\cdot, u, \lambda), K g(\cdot, u, \lambda)\rangle+\lambda\langle K(I-P) h, g(\cdot, u, \lambda)\rangle \\
\leq & \langle Q g(\cdot, u, \lambda), K Q g(\cdot, u, \lambda)\rangle+\langle(I-Q) g(\cdot, u, \lambda), K(I-Q) g(\cdot, u, \lambda)\rangle \\
& +\lambda\langle K(I-P) h, g(\cdot, u, \lambda)\rangle \\
\leq & (2 N+1)^{-1}|Q g(\cdot, u, \lambda)|_{2}^{2}+(4 N+4)^{-1}|(I-Q) g(\cdot, u, \lambda)|_{2}^{2} \\
& +|K(I-P) h|_{2}\left(a|u|_{2}+b\right),
\end{aligned}
$$

because

$$
\sup \left\{\langle w, K(I-Q) w\rangle: P w=0 \text { and }|w|_{2}=1\right\}=(4 N+4)^{-1} .
$$

Hence, we can conclude that

$$
\text { for every } \varepsilon>0 \text {, there exists } r_{\varepsilon} \text { such that }
$$

$$
|(I-Q) g(\cdot, u, \lambda)|_{2} \leq \varepsilon|u|_{2}+r_{\varepsilon} .
$$

Now we rewrite equation (2.4) in the form

$$
u-P u=K g(\cdot, u, \lambda)+\lambda K(I-P) h .
$$

Applying the projection $Q$ and using again $K Q=Q K$, we find

$$
Q u=K Q g(\cdot, u, \lambda)+\lambda K Q h .
$$

Subtraction then gives

$$
u-P u-Q u=K(I-Q) g(\cdot, u, \lambda)+\lambda K(I-P-Q) h .
$$

Finally, (2.7) and (2.8) yield (2.3), by the boundedness of $K$.

Step 2. We prove that $\left(f_{1}\right)$ implies the estimate

$$
\text { for every } \varepsilon>0 \text {, there exists } k_{\varepsilon} \text { such that if }|u|_{\infty} \geq k_{\varepsilon} \text { then either }
$$

$$
\|u-P u\| \leq \varepsilon|u|_{\infty} \text { or }\|u-Q u\| \leq \varepsilon|u|_{\infty} .
$$

Assume, by contradiction, the existence of a sequence $\left\{u_{n}\right\}$ of solutions of (2.2), for $\lambda=\lambda_{n} \in[0,1]$, with $\left|u_{n}\right|_{\infty} \rightarrow+\infty$, such that 
From the equation in (2.2), we derive that, possibly passing to a subsequence, $\left\{p_{n}\right\}$ converges in $L^{1}(0,2 \pi)$ and a.e. in $[0,2 \pi]$ to some $p \in L^{1}(0,2 \pi)$. This function $p$ can be written in the form $p=m v$, with $m \in L^{\infty}(0,2 \pi)$ satisfying

$$
N^{2} \leq m(t) \leq(N+1)^{2},
$$

for a.e. $t \in[0,2 \pi]$. Indeed, let $t$ be such that $p_{n}(t) \rightarrow p(t)$. If $v(t) \neq 0$ then $\left|u_{n}(t)\right| \rightarrow+\infty$. Hence, for any fixed $\delta>0$, we get by $\left(f_{1}\right)$

$$
N^{2}-\delta \leq p_{n}(t)\left|u_{n}\right|_{\infty} / u_{n}(t) \leq(N+1)^{2}+\delta
$$

for all large $n$ and, therefore, passing to the limits

$$
N^{2} \leq p(t) / v(t) \leq(N+1)^{2} .
$$

Finally, observing that $v$ vanishes on a set of zero measure, we can set $m(t)=$ $p(t) / v(t)$ for a.e. $t \in[0,2 \pi]$ and conclude that $m$ is measurable and satisfies (2.10).

Accordingly, $v$ is a $2 \pi$-periodic solution of the equation $-v^{\prime \prime}=m v$. Hence, recalling that $v=\varphi+\psi$, we have

$$
N^{2} \varphi+(N+1)^{2} \psi=m \varphi+m \psi .
$$

Multiplying this relation by $\varphi$ and $\psi$, respectively, and integrating on $[0,2 \pi]$, by $(2.10)$, we get

$$
0 \leq \int_{[0,2 \pi]}\left(m-N^{2}\right) \varphi^{2}=-\int_{[0,2 \pi]} m \varphi \psi=\int_{[0,2 \pi]}\left(m-(N+1)^{2}\right) \psi^{2} \leq 0,
$$

which yields

$$
\int_{[0,2 \pi]}\left(m-N^{2}\right) \varphi^{2}=0=\int_{[0,2 \pi]}\left((N+1)^{2}-m\right) \psi^{2} .
$$

Using the analyticity of $\varphi$ and $\psi$ and the fact that either $\varphi \not \equiv 0$ or $\psi \not \equiv 0$, we conclude that either $m \equiv N^{2}$ and $\psi \equiv 0$ or $m \equiv(N+1)^{2}$ and $\varphi \equiv 0$, a.e. in $[0,2 \pi]$. This finally implies that

$$
(I-P) u_{n} /\left|u_{n}\right|_{\infty} \rightarrow(I-P) \varphi=0
$$

or

$$
(I-Q) u_{n} /\left|u_{n}\right|_{\infty} \rightarrow(I-Q) \psi=0
$$

in $W^{2,1}(0,2 \pi)$. Thus a contradiction is reached.

Step 3. We prove that $\left(f_{1}\right)$ and $\left(F_{1}\right)$ imply that

there exist a sequence $\left\{R_{n}\right\}$, with $R_{n} \rightarrow+\infty$, and an integer $n_{0}$ such that $\max u \neq R_{n}$,

for every $n>n_{0}$.

Let us set

$$
\begin{aligned}
& g_{1}(s, \lambda)=(1-\lambda) \vartheta s+\lambda f(s)-N^{2} s, \\
& g_{2}(s, \lambda)=(1-\lambda) \vartheta s+\lambda f(s)-(N+1)^{2} s
\end{aligned}
$$


for $s \in \mathbb{R}$ and $\lambda \in[0,1]$ and denote by

$$
G_{1}(s, \lambda)=\int_{[0, s]} g_{1}(\xi, \lambda) d \xi, \quad G_{2}(s, \lambda)=\int_{[0, s]} g_{2}(\xi, \lambda) d \xi
$$

the respective primitives. A solution $u$ of (2.2) is then a solution of both equations

$$
\begin{aligned}
-u^{\prime \prime}-N^{2} u & =g_{1}(u, \lambda)+\lambda h, \\
-u^{\prime \prime}-(N+1)^{2} u & =g_{2}(u, \lambda)+\lambda h .
\end{aligned}
$$

Claim 1. For every $\varepsilon>0$, there exists $k_{\varepsilon}$ such that if $|u|_{\infty} \geq k_{\varepsilon}$ then either

$$
\left|g_{1}(u, \lambda)\right|_{1} \leq c\left(\varepsilon|u|_{\infty}+1\right) \text { or }\left|g_{2}(u, \lambda)\right|_{1} \leq c\left(\varepsilon|u|_{\infty}+1\right),
$$

where $c$ is a constant independent of $u, \lambda$, and $\varepsilon$.

Indeed, fix $\varepsilon>0$, take $u$ such that $|u|_{\infty} \geq k_{\varepsilon}$, and suppose that (cf. (2.9)) $\|u-P u\| \leq \varepsilon|u|_{\infty}$ (in the other case, the proof would be similar). By equation (2.11), using the boundedness of the operator $-d^{2} / d t^{2}-N^{2} I$ from $W^{2,1}(0,2 \pi)$ to $L^{1}(0,2 \pi)$, we get, for some constant $c$,

$$
\left|g_{1}(u, \lambda)\right|_{1}=\left|-u^{\prime \prime}-N^{2} u-\lambda h\right|_{1} \leq c(\|u-P u\|+1) \leq c\left(\varepsilon|u|_{\infty}+1\right) .
$$

Hence, Claim 1 is proved.

Claim 2. There are constants $k_{1}$ and $d$ (independent of $u$ and $\lambda$ ) such that if $|u|_{\infty} \geq k_{1}$ then $\left|u^{\prime}\right|_{\infty} \leq d|u|_{\infty}$.

Indeed, take $u$ such that $|u|_{\infty} \geq k_{1}$, where $k_{1}$ is given by (2.9) for the choice $\varepsilon=1$, and assume that $\|u-P u\| \leq|u|_{\infty}$ (similar proof in the other case). Hence, we have, for some constant $d$,

$$
\left|u^{\prime}\right|_{\infty} \leq\left|(P u)^{\prime}\right|_{\infty}+\left|(u-P u)^{\prime}\right|_{\infty} \leq N|P u|_{\infty}+\left|(u-P u)^{\prime \prime}\right|_{1} \leq d|u|_{\infty} .
$$

Thus, Claim 2 is proved.

Claim 3. There are constants $k_{2}$ and $c_{1}, c_{2}$ (independent of $u$ and $\lambda$ ), with $0<c_{1}<1<c_{2}$, such that if $|u|_{\infty} \geq k_{2}$ then

$$
\min u<0<\max u \quad \text { and } \quad c_{1} \leq \max u /-\min u \leq c_{2} \text {. }
$$

Indeed, let $\left\{u_{n}\right\}$ be any sequence of solutions of $(2.2)$, for $\lambda=\lambda_{n} \in[0,1]$, such that $\left|u_{n}\right|_{\infty} \rightarrow+\infty$. We know (cf. Step 2) that either

$$
u_{n} /\left|u_{n}\right|_{\infty} \rightarrow \varphi, \quad \text { with } \varphi \in \operatorname{Im} P \text { and }|\varphi|_{\infty}=1
$$

or

$$
u_{n} /\left|u_{n}\right|_{\infty} \rightarrow \psi, \quad \text { with } \psi \in \operatorname{Im} Q \text { and }|\psi|_{\infty}=1
$$

in $W^{2,1}(0,2 \pi)$ and then uniformly on $[0,2 \pi]$. Accordingly, we have that either

$$
\max u_{n} /\left|u_{n}\right|_{\infty} \rightarrow \max \varphi \text { and } \min u_{n} /\left|u_{n}\right|_{\infty} \rightarrow \min \varphi
$$

or

$$
\max u_{n} /\left|u_{n}\right|_{\infty} \rightarrow \max \psi \quad \text { and } \min u_{n} /\left|u_{n}\right|_{\infty} \rightarrow \min \psi
$$

In any case, from $\max \varphi /-\min \varphi=1=\max \psi /-\min \psi$, we get

$$
\max u_{n} /-\min u_{n} \rightarrow 1 \text {. }
$$

Hence, Claim 3 follows arguing by contradiction. 
Note that for proving Claims 1 and 2 only assumption $\left(f_{1}\right)$ has been used, while in the proof of Claim 3 the oscillatory properties of the eigenfunctions have been exploited as well.

We now observe that $\left(\mathrm{F}_{1}\right)$ and the continuity of $F(s) / s^{2}$, for $s \neq 0$, imply that

there exists a sequence $\left\{R_{n}\right\}$, with $R_{n} \rightarrow+\infty$, such that

Using $\left(F_{1}^{\prime}\right)$ and $\left(f_{1}\right)$, we then prove that

there exists an integer $n_{0}$ such that, for every $n>n_{0}$,

$$
\max u \neq R_{n} \text {. }
$$

Under the above positions, $\left(F_{1}^{\prime}\right)$ implies that there are constants $\varepsilon_{1}, \varepsilon_{2}>0$ such that

$$
\lim _{n \rightarrow+\infty} G_{1}\left(R_{n}, \lambda\right) / R_{n}^{2} \geq \varepsilon_{1} \text { and } \lim _{n \rightarrow+\infty} G_{2}\left(R_{n}, \lambda\right) / R_{n}^{2} \leq-\varepsilon_{2},
$$

uniformly in $\lambda \in[0,1]$. Take $\varepsilon>0$ such that

$$
\varepsilon<\min \left\{\varepsilon_{1} c_{1}^{2} /(2 c d), \varepsilon_{2} c_{1}^{2} /(2 c d)\right\},
$$

with $c, d, c_{1}$ given by Claims 1,2 , and 3 , and let $u$ be a solution such that

$$
|u|_{\infty} \geq k=\max \left\{k_{\varepsilon}, k_{1}, k_{2}\right\},
$$

with $k_{\varepsilon}, k_{1}, k_{2}$ given by Claims 1,2 , and 3. Moreover, suppose that in Claim 1 , it is

$$
\left|g_{1}(u, \lambda)\right|_{1} \leq c\left(\varepsilon|u|_{\infty}+1\right)
$$

(similar proof in the other case). Let $t_{1}, t_{2} \in[0,2 \pi]$ be such that $u\left(t_{2}\right)=\max u$ and $u\left(t_{1}\right)=0\left(t_{1}\right.$ does exist, since Claim 3 implies that $u$ changes sign). Using Claims 2 and 3 , we easily get

$$
\begin{aligned}
G_{1}(\max u, \lambda) & =G_{1}\left(u\left(t_{2}\right), \lambda\right)-G_{1}\left(u\left(t_{1}\right), \lambda\right)=\int_{\left[t_{1}, t_{2}\right]} g_{1}(u(\xi), \lambda) u^{\prime}(\xi) d \xi \\
& \leq\left|g_{1}(u, \lambda)\right|_{1}\left|u^{\prime}\right|_{\infty} \leq c d\left(\varepsilon|u|_{\infty}+1\right)|u|_{\infty} \\
& \leq \varepsilon\left(c d / c_{1}^{2}\right)(\max u)^{2}+\left(c d / c_{1}\right) \max u .
\end{aligned}
$$

Let $n_{0}$ be an integer such that, for every $n>n_{0}$,

$$
G_{1}\left(R_{n}, \lambda\right) \geq\left(\varepsilon_{1} / 2\right) R_{n}^{2}
$$

for every $\lambda \in[0,1]$ and

$$
R_{n}>\max \left\{k,\left(\left(\varepsilon_{1} / 2\right)-\varepsilon\left(c d / c_{1}^{2}\right)\right)^{-1}\left(c d / c_{1}\right)\right\} .
$$

Hence, we easily conclude that $\max u \neq R_{n}$, for every $n>n_{0}$.

Step 4. We prove that $\left(f_{1}\right)$ and $\left(F_{1}^{\prime}\right)$ imply the existence of a solution of problem (1.1).

Claim 4. For every $A$ ( $>k_{2}$, with $k_{2}$ defined in Claim 3), there exists $B$ $\left(>k_{2}\right)$ such that if $\max u \leq A$ then $\min u>-B$. 
Indeed, by Claim 3, we know that, whenever $|u|_{\infty} \geq k_{2}$,

$$
\min u<0<\max u \quad \text { and } \quad c_{1} \leq \max u /-\min u \leq c_{2} .
$$

Hence, taking any $B>A / c_{1}\left(>k_{2}\right)$, we have that if $|u|_{\infty} \geq k_{2}$ and $\max u \leq A$ then

$$
\min u \geq-A / c_{1}>-B .
$$

Whereas, if $|u|_{\infty}<k_{2} \quad(<\min \{A, B\})$ then

$$
-B<\min u \leq \max u<A .
$$

Thus, Claim 4 is proved.

By Step 3 and Claim 4, we derive that, taking $A=R_{n}$, for any $n>n_{0}$, there is no solution $u$ of (2.2), with $-B \leq u(t) \leq A$, for every $t \in[0,2 \pi]$, such that $\max u=A$ or $\min u=-B$. Now let us define in $C^{0}([0,2 \pi])$ the following open bounded set, containing 0 ,

$$
\Omega=\left\{u \in C^{0}([0,2 \pi]):-B<u(t)<A \text { for every } t \in[0,2 \pi]\right\} .
$$

Since no solution $u \in \operatorname{cl} \Omega$ of (2.2) for some $\lambda \in[0,1]$ belongs to bdry $\Omega$, we can conclude that equation (2.1), and therefore problem (1.1), has at least one solution $u \in \Omega$ according to the homotopy invariance of the degree.

Step 5. We prove that $\left(f_{1}\right)$ and $\left(F_{3}\right)$ imply the existence of a solution of problem (1.1).

At first we note that two situations may occur:

$$
\liminf _{s \rightarrow+\infty} 2 F(s) / s^{2}<(N+1)^{2}
$$

or

$$
\liminf _{s \rightarrow+\infty} 2 F(s) / s^{2}=(N+1)^{2}>N^{2} .
$$

If $\left(F_{3}^{\prime}\right)$ holds then $\left(F_{3}\right)$ implies $\left(F_{1}\right)$, and therefore the existence of a solution of problem (1.1) follows from the previous steps. Accordingly, we have to prove the solvability under $\left(f_{1}\right),\left(F_{3}^{\prime \prime}\right)$, and

$$
\liminf _{s \rightarrow-\infty} 2 F(s) / s^{2}<(N+1)^{2} .
$$

Clearly, $\left(\mathrm{F}_{3}^{\prime \prime}\right)$ and $\left(\mathrm{F}_{3}^{\prime \prime \prime}\right)$ yield the following conditions for $G_{1}(s, \lambda)$ and $G_{2}(s, \lambda)$, respectively,

$$
\liminf _{s \rightarrow+\infty} G_{1}(s, \lambda) / s^{2} \geq \varepsilon_{1}>0
$$

and for some sequence $\left\{R_{n}\right\}$ with $R_{n} \rightarrow+\infty$

$$
\lim _{n \rightarrow+\infty} G_{2}\left(-R_{n}, \lambda\right) / R_{n}^{2} \leq-\varepsilon_{2}<0,
$$

where both limits are uniform in $\lambda \in[0,1]$. Take $\varepsilon>0$ such that

$$
\varepsilon<\min \left\{\varepsilon_{1} c_{1}^{2} /(2 c d), \varepsilon_{2} /\left(2 c d c_{2}^{2}\right)\right\},
$$

with $c, d, c_{1}, c_{2}$ given by Claims 1,2 , and 3 , and let $u$ be a solution such that

$$
|u|_{\infty} \geq k=\max \left\{k_{\varepsilon}, k_{1}, k_{2}\right\}
$$


with $k_{\varepsilon}, k_{1}, k_{2}$ given by Claims 1,2 , and 3 . Now we distinguish between two possibilities:

$$
\left|g_{1}(u, \lambda)\right|_{1} \leq c\left(\varepsilon|u|_{\infty}+1\right)
$$

or

$$
\left|g_{2}(u, \lambda)\right|_{1} \leq c\left(\varepsilon|u|_{\infty}+1\right) .
$$

Assume that (2.13) holds. Let $t_{1}$ and $t_{2}$ be chosen as in Step 3. Proceeding as in Step 3, we obtain

$$
G_{1}(\max u, \lambda) \leq \varepsilon\left(c d / c_{1}^{2}\right)(\max u)^{2}+\left(c d / c_{1}\right) \max u .
$$

Hence, it is clear that $\max u<R$, for any $R>0$ such that

$$
G_{1}(s, \lambda) \geq\left(\varepsilon_{1} / 2\right) s^{2}
$$

for every $s \geq R$ and $\lambda \in[0,1]$, and

$$
R>\max \left\{k,\left(\left(\varepsilon_{1} / 2\right)-\varepsilon\left(c d / c_{1}^{2}\right)\right)^{-1}\left(c d / c_{1}\right)\right\} .
$$

Assume now that $(2.14)$ holds. Let $t_{1}, t_{2} \in[0,2 \pi]$ be such that $u\left(t_{2}\right)=\min u$ and $u\left(t_{1}\right)=0$. Proceeding as in Step 3, we get

$$
\begin{aligned}
G_{2}(\min u, \lambda) & =G_{2}\left(u\left(t_{2}\right), \lambda\right)-G_{2}\left(u\left(t_{1}\right), \lambda\right)=\int_{\left[t_{1}, t_{2}\right]} g_{2}(u(\xi), \lambda) u^{\prime}(\xi) d \xi \\
& \geq-\left|g_{2}(u, \lambda)\right|_{1}\left|u^{\prime}\right|_{\infty} \geq-c d\left(\varepsilon|u|_{\infty}+1\right)|u|_{\infty} \\
& \geq-\varepsilon\left(c d c_{2}^{2}\right)(\min u)^{2}+\left(c d c_{2}\right) \min u .
\end{aligned}
$$

Let $n_{0}$ be an integer such that for every $n>n_{0}$

$$
G_{2}\left(-R_{n}, \lambda\right) \leq-\left(\varepsilon_{2} / 2\right) R_{n}^{2}
$$

for every $\lambda \in[0,1]$ and

$$
R_{n}>\max \left\{k,\left(\left(\varepsilon_{2} / 2\right)-\varepsilon\left(c d c_{2}^{2}\right)\right)^{-1}\left(c d c_{2}\right)\right\} .
$$

Hence, we can conclude that $\min u \neq-R_{n}$ for every $n>n_{0}$.

Finally, we are in position to prove the existence of a solution of problem (1.1). Take an integer $n>n_{0}$ such that $R_{n} \geq R / c_{1}$, and, as in Step 4, define the open set

$$
\Omega=\left\{u \in C^{0}([0,2 \pi]):-B<u(t)<A \quad \text { for every } t \in[0,2 \pi]\right\},
$$

where now $B=R_{n}$ and $A=c_{2} B \quad(>R)$. Let $u$ be a solution of (2.2) belonging to $\mathrm{cl} \Omega$. Clearly, $u$ belongs to $\Omega$ if $|u|_{\infty}<k \quad(<\min \{A, B\})$. Therefore, suppose that $|u|_{\infty} \geq k$. By Claim 3, we have that if (2.13) holds, $\max u<R<A$ and then $\min u>-R / c_{1} \geq-B$. On the other hand, if (2.14) holds, $\min u>-B$ and then $\max u<c_{2} B=A$. Accordingly, such a solution $u$ belongs to $\Omega$. The solvability of problem (1.1) then follows as in Step 4 . Thus the proof is concluded. 


\section{REFERENCES}

[ALL] S. Ahmad, A. C. Lazer, and J. L. Paul, Elementary critical point theory and perturbations of elliptic boundary value problems at resonance, Indiana Univ. Math. J. 25 (1976), 933-944.

[CO] D. Costa and A. Oliveira, Existence of solutions for a class of semilinear elliptic problems at double resonance, Boll. Soc. Brasil Mat. 19 (1988), 21-37.

[DFG] D. G. De Figueiredo and J. P. Gossez, Conditions de non-résonance pour certains problèmes elliptiques semi-linéaires, C. R. Acad. Sci. Paris 302 (1986), 543-545.

[D] T. Ding, Nonlinear oscillations at a point of resonance, Sci. Sinica Ser. A 25 (1982), 918-931.

[DIZ] T. Ding, R. Iannacci, and F. Zanolin, Existence and multiplicity results for periodic solutions of semilinear Duffing equations, preprint, 1990.

[DZ] T. Ding and F. Zanolin, Time-maps for the solvability of periodically perturbed nonlinear Duffing equations, Nonlinear Anal. T.M.A. (to appear).

[FF] C. Fabry and A. Fonda, Periodic solutions of nonlinear differential equations with double resonance, Ann. Mat. Pura Appl. 157 (1990), 99-116.

[GO1] J. P. Gossez and P. Omari, Nonresonance with respect to the Fucik spectrum for periodic solutions of second order ordinary differential equations, Nonlinear Anal. T.M.A. 14 (1990), 1079-1104.

[GO2] __ Periodic solutions of a second order ordinary differential equation: a necessary and sufficient condition for nonresonance, J. Differential Equations 94 (1991), 67-82.

[MW1] J. Mawhin and M. Willem, Critical points of convex perturbations of some indefinite quadratic forms and semilinear boundary value problems at resonance, Ann. Inst. Henri Poincaré 6 (1986), 431-453.

[MW2] _ Critical Point Theory and Hamiltonian systems, Springer-Verlag, New York, 1989.

[OZ] P. Omari and F. Zanolin, A note on nonlinear oscillations at resonance, Acta Math. Sinica (N.S.) 3 (1987), 351-361.

[Q] D. Qian, An abundance of periodic solutions for Duffing equation with oscillatory time-map, preprint, 1990.

[R] M. Ramos, Remarks on resonance problems with unbounded perturbations, preprint, 1990.

Dipartimento di Scienze Matematiche, Università di Trieste, Piazzale Europa 1, 34127 TRIESTE, ITALIA

Dipartimento di Matematica e Informatica, Università di Udine, Via Zanon 6, 33100 Udine, ITALiA 